\title{
NUEVA LOCALIDAD GEOGRAFICA PARA BERBERIS NEGERIANA TISCHLER (BERBERIDACEAE) EN LA PROVINCIA DE ARAUCO, REGION DEL BIO BIO
}

\author{
NEW GEOGRAPHICAL LOCALITY FOR BERBERIS NEGERIANA TISCHLER \\ (BERBERIDACEAE) IN THE PROVINCE OF ARAUCO, BIO BIO REGION
}

\author{
Persy Gómez 1 , Michail Belov² \& José San Martín ${ }^{3}$ \\ ${ }^{1}$ Jardín Botánico de la Universidad de Talca, Casilla 747, Talca, Chile; 2Chile Flora, Hijuela \# 2, Lihueno, Pelarco, \\ Talca, Chile; 3 Instituto de Biología Vegetal y Biotecnología de la Universidad de Talca, Casilla 747, Talca, Chile. \\ jbotanico@utalca.cl
}

\begin{abstract}
This note reports the finding of a population of Berberis negeriana Tischler (Berberidaceae), in the province of Arauco, Bio Bio Region ( $37^{\circ} 42^{\prime} 17^{\prime \prime} S$ y $\left.73^{\circ} 19^{\prime} 43^{\prime \prime} \mathrm{W}\right)$ in the center south of Chile. The population was found between Nothofagus obliqua and species of the Valdiviano Forest.
\end{abstract}

En su distribución mundial la familia Berberidaceae incluye 15 géneros y 650 especies con dos centros de diversidad (Loconte 1993). Para el hemisferio norte, específicamente Eurasia, está presente el Subgénero Septentrional C.K.Schneid. que incluye aproximadamente 300 especies, de las cuales 100 están restringidas al área señalada. Para el hemisferio sur, concretamente Sudamérica templada y andina, se encuentra el Subgénero Austral C.K.Schneid, con alrededor de 200 especies. Para Chile continental, la familia está representada por 16 especies nativas del género Berberis (Landrum 2003).

El material recolectado fue comparado con la descripción de Landrum (2003), y posteriormente confirmado por comparación con material depositado en el herbario del Museo Nacional de Historia Natural (SGO) y en el herbario de Concepción (CONC).

En el sitio, la población de B. negeriana comprende sólo cuatro individuos en una quebrada poco profunda, en el límite de un bosque de Nothofagus obliqua (Mirb.) Oerst. y otro remanente de bosque tipo valdiviano. Uno de los individuos se encuentra en fase juvenil y los restantes en estado adulto con altura promedio de $2,5 \mathrm{~m}$, superando la reportada por Landrum (2003). En el mes de agosto, algunos individuos se encontraban en el inicio del estado de flora- ción, presentando una apariencia saludable (Figuras 1 A y B) y con ausencia de daños por el ganado doméstico.

Entre las especies acompañantes a B. negeriana están Aextoxicon punctatum Ruiz et Pav., Gomortega keule (Molina) Baill., Lomatia dentata (Ruiz et Pav.) R.Br., Laurelia sempervirens (Ruiz et Pav.) Tul. y Rhaphithamnus spinosus (Juss.) Mold.

Antecedentes orales de lugareños locales dan cuenta de la existencia de otras dos poblaciones de $B$. negeriana con aproximadamente la misma cantidad de individuos. Dicha información necesita ser cotejada en una nueva excursión.

En Chile la distribución de B. negeriana estaba restringida a solamente dos poblaciones, una situada en la provincia de Concepción, Chiguayante-Nonguén $\left(36^{\circ} 50^{\prime} \mathrm{S}\right)$ y la otra en la provincia de Arauco ( $37^{\circ} 20^{\prime}$ S), en la caleta Yani (Hechenleitner et al. 2005). Este nuevo registro de Berberis negeriana, al sur del Río Caramávida, representa una ampliación del rango de distribución conocido para la especie en aproximadamente $70 \mathrm{Km}$ hacia el sur. Desde el punto de vista de la conservación la especie no está protegida y en su medio natural las amenazas se dan en su hábitat con tala de la vegetación arbórea y sustitución por plantaciones forestales (Hechenleitner et al. 2005). 

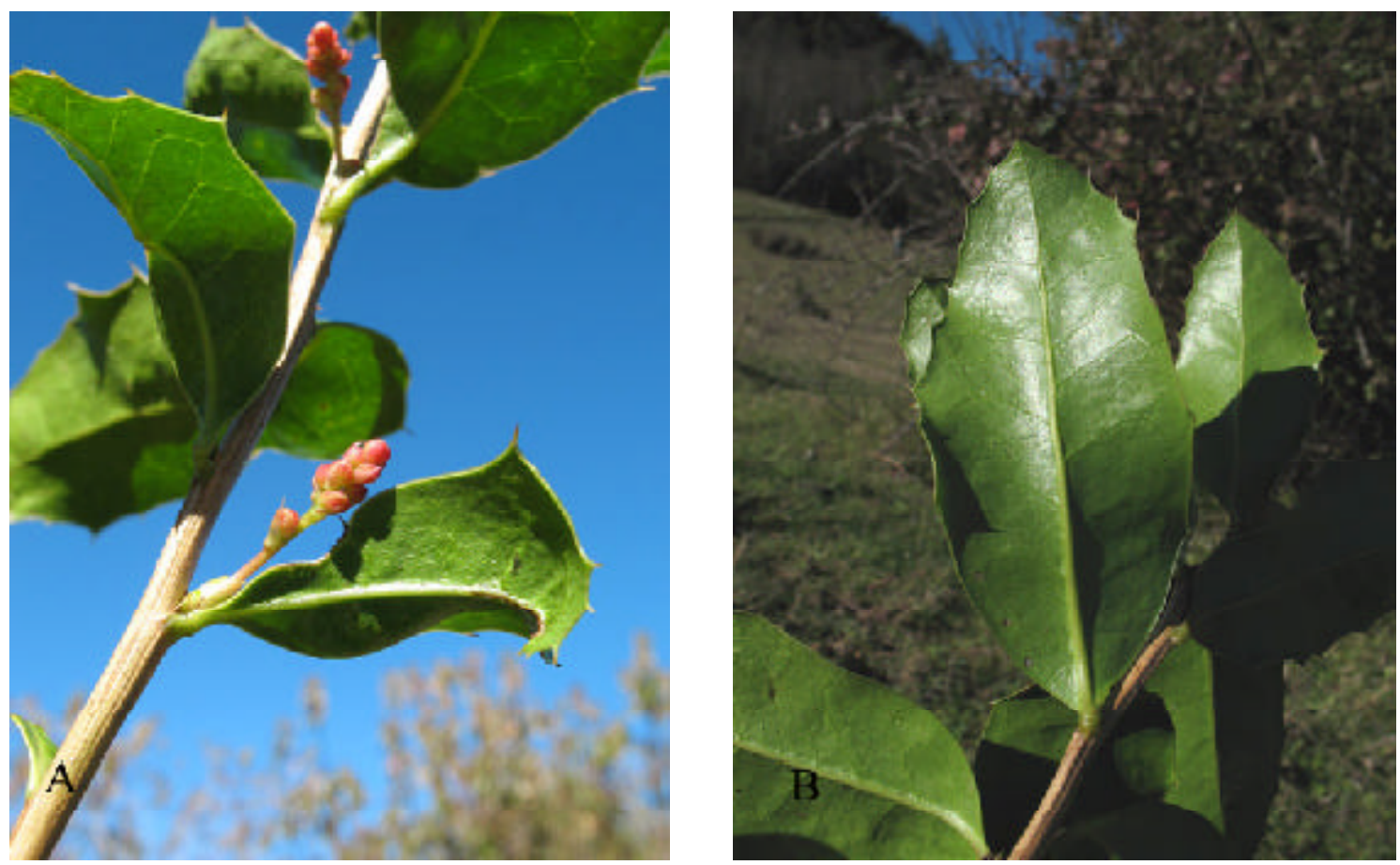

Figura 1. Berberis negeriana Tischler. A. Envés de la hoja. B. Tallos con hojas e inflorescencias axilares en antesis (fotografias de Michail Belov).

Figure 1. Berberis negeriana Tischler. A. Instead of the leaf. B. Branch with leaves and axillars inflorescences in anthesis (photographs by Michail Belov).

MATERIAL ESTUDIADO: VIII Región: Prov. Concepción. Chiguayante, hills $\mathrm{N}$ of town, climbed ca. 1 hour. 30-XI-1993. L.R Landrum \& E. Ruiz 8210 (SGO 132623); Bosque Fisco at Nonguén, SE of Concepción (36 $50^{\circ} \mathrm{S}, 7^{\circ} 02^{\prime} \mathrm{W}$ ) beyond houses, ca. 300-500 m. 16-XII-1994. L.R Landrum \& E. Ruiz. 8385 (SGO 134367); Concepción, en los cerros ConcepciónNonguén, 130 m. 25-X-1952. M. Ricardi. (CONC 13215); Prov. Arauco, comuna Los Álamos, sur del Río Caramávida, aprox. $7 \mathrm{~km}$ al SE desde Antiguala y 3 km desde Hostal Caramávida (37²4'17" S, 7319'43" W), 300 m. 4-VIII-2007. M. Belov (Universidad de Talca, $\mathrm{N}^{\circ} 3142$ ).

\section{AGRADECIMIENTOS}

Se agradece la disposición de la Sra. Mélica Muñoz y Sra. Inés Mesa, del Museo Nacional de Historia
Natural de Santiago (SGO), y a Alicia Marticorena del herbario de la Universidad de Concepción (CONC) por facilitar la consulta de los ejemplares revisados.

\section{BIBLIOGRAFIA}

Hechenleitner, V., P., M. F. Gardner, P. I. Thomas, C. Echeverria, B. Escobar, P.Brownless \& C. Martínez. 2005. Plantas Amenazadas del Centro-Sur de Chile. Distribución, Conservación y Propagación. Primera Edición. Universidad Austral de Chile y Real Jardín Botánico de Edimburgo. $188 \mathrm{pp}$.

LANDRum, L. 2003. Berberidaceae. En: Marticorena C. \& R. Rodríguez (eds.). Flora de Chile. Vol. 2(2):18.Universidad de Concepción, Concepción, Chile. 93 pp.

Loconte, H. 1993. Berberidaceae, in K. Kubitzki (ed.), The families and genera of vascular plants 2: 147152.

Recibido:

Aceptado: 